\title{
Evolution of source characteristics of AE events during frictional sliding
}

\author{
Yasuo Yabe \\ Research Center for Prediction of Earthquakes and Volcanic Eruptions, Graduate School of Science, Tohoku University
}

(Received March 17, 2008; Revised May 14, 2008; Accepted May 30, 2008; Online published June 18, 2008)

\begin{abstract}
The evolution of source characteristics of acoustic emission (AE) events and the surface topography of the fault surface during frictional sliding were investigated with the aim of improving current understanding of the microscopic process of friction. Both the AE source dimensions and the grain-scale topographies were well conserved against abrasion during the sliding, but the magnitude of stress drop was significantly reduced. The fractal-domain topographies, the wavelength of which is shorter than the fractal limit wavelength $\left(\lambda_{c}\right)$, were significantly worn during the sliding. These results suggest that the grain-scale topography determined the AE source dimension, while the fractal-domain asperities controlled the magnitude of the stress drop. Since elastic wave radiation is one of the major energy consumption processes, the grain-scale and the fractal-domain topographies may play different roles, not only in the AE source process but also in the friction of rocks.
\end{abstract}

Key words: Acoustic emission, rock friction, evolution of AE, fault surface evolution.

\section{Introduction}

Acoustic emission (AE) events have been observed during frictional sliding on pre-cut faults in laboratory rock samples (e.g., Weeks et al., 1978; Sammonds and Ohnaka, 1998; Yabe et al., 2003). The AE sources were located on the pre-cut fault, and their focal mechanism solution was consistent with that expected theoretically for macroscopic sliding. An AE event during frictional sliding should be generated by unstable slip on a small part of a pre-cut fault on which the macroscopic stable-sliding takes place. Yabe (2002) showed that the statistical characteristics of AE activity are strongly related to the frictional properties of fault. It is generally considered that energy supplied by the testing machine to maintain fictional sliding is consumed by the elastic wave radiation, the heat generation and the surface energy to generate wear gouges and fracture surfaces. Yoshioka (1986) estimated the proportion of surface energy to total energy consumed by frictional sliding and found that the surface energy occupies only $0.1-0.01 \%$ of the total energy. It can therefore be considered that most part of the frictional energy is consumed by the elastic wave radiation and the heat generation. As a result, the AE source process should be strongly related to the microscopic processes of friction. Although the source parameters of $\mathrm{AE}$ events generated during the failure of intact rocks was examined in detail by Seller et al. (2003), the source process of $\mathrm{AE}$ events accompanying the frictional sliding has not yet been investigated. In this paper, the evolution of $\mathrm{AE}$ source characteristics and fault surface topography associated with accumulated sliding is examined to elucidate the occurrence process of $\mathrm{AE}$ events during sliding.

Copy right (C) The Society of Geomagnetism and Earth, Planetary and Space Sciences (SGEPSS); The Seismological Society of Japan; The Volcanological Society of Japan; The Geodetic Society of Japan; The Japanese Society for Planetary Sciences; TERRAPUB.

\section{Experimental Procedure}

Since the experimental procedure was the same as that of Yabe et al. (2003), it is only described briefly here. An Oshima granite specimen, $300 \times 300 \times 100 \mathrm{~mm}^{3}$, with a diagonally pre-cut fault $400 \mathrm{~mm}$ in length (Fig. 1(a)) was biaxially compressed with a servo-controlled loading apparatus. The fault surfaces were prepared using a \#60 abrasive with a mean particle size of $250 \mu \mathrm{m}$. The topographies of the ground surfaces of the pre-cut fault were measured along nine profiles, each $200 \mathrm{~mm}$ long, on each surface before and after the sliding of about $17 \mathrm{~mm}$. The applied normal stress was $10 \mathrm{MPa}$ and kept constant within an error of $0.5 \mathrm{MPa}$. The average ram stroke rate was $0.45 \mu \mathrm{m} / \mathrm{s}$, which corresponds to the macroscopic sliding rate of $0.64 \mu \mathrm{m} / \mathrm{s}$.

AE events were observed by 11 piezoelectric transducers made of PZT ceramics. Seven of these were broadbandtype transducers (NF AE-900F1), two were resonant-type transducers with a resonance frequency of $2 \mathrm{MHz}$ and other two were shear-mode transducers. The sensitivity of the broadband-type transducer for ground velocity is $398 \mathrm{~V} /(\mathrm{m} / \mathrm{s})$ and approximately flat $( \pm 3 \mathrm{~dB})$ in the frequency range between $200 \mathrm{kHz}$ and $1.3 \mathrm{MHz}$. When the output amplitude of PZT4 exceeded a prescribed threshold level, the wave-memorizers were triggered to record the AE waveforms at a sampling frequency of $5 \mathrm{MHz}$. The record length was $819.2 \mu \mathrm{s}$ for each channel, with a pre-trigger delay of $204.8 \mu \mathrm{s}$.

\section{Analyses and Results}

\subsection{Source characteristics of AE}

The sliding mode of the fault was stable sliding, with the exception of sliding from 6.5 to $12 \mathrm{~mm}$, for which the macroscopic sliding mode was episodic sliding. Since Yabe (2002) was able to demonstrate that the statistical characteristics of AE depends on the sliding rate, I excluded the events during episodic sliding from subsequent analyses to 
(a)
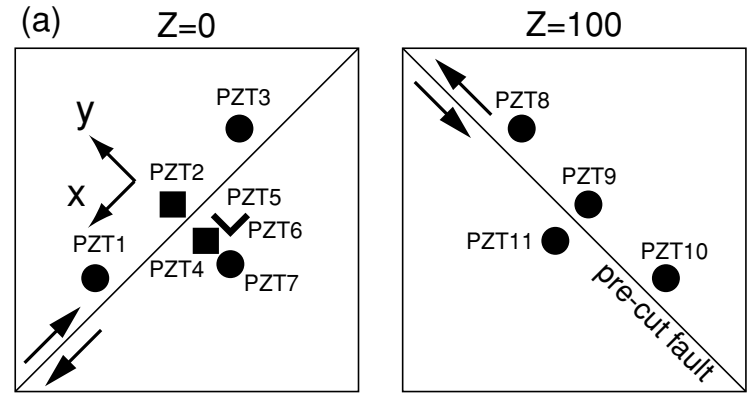

(b)

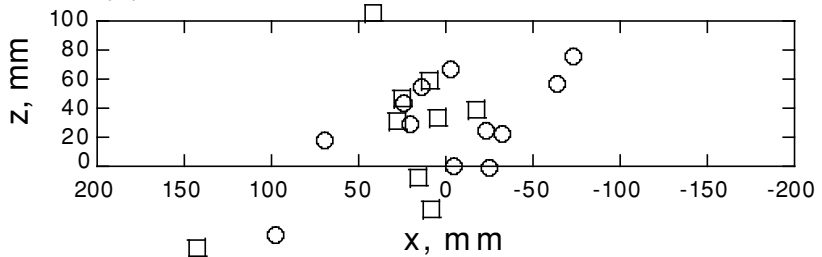

Fig. 1. (a) Sensor distribution on both sides of rock sample. A Cartesian coordinate system $(x, y, z)$ is defined for the AE hypocenter determination. The fault plane is $y=0$. The $x$-axis is taken along the fault strike, and the $z$-axis is taken in the direction of the specimen thickness; the free surfaces of the specimen are $z=0,100 \mathrm{~mm}$. The origin of the coordinate system is taken at the center of the $z=0$ surface. Solid circles and solid squares represent the broadband-type transducers and the resonance-type ones, respectively. PZT5 and PZT6 were resonance-type transducers of shear-mode, where the direction of a line-segment indicates the most sensitive motion direction of the transducer. The diagonal lines show the pre-cut fault trace. Paired-arrows indicate the sliding direction of individual blocks. (b) Hypocenter distribution of the small-sliding events (open circles) and the large-sliding ones (open squares).

avoid the effect of sliding rate overlaying the evolution of AE occurrence with sliding distance. The friction coefficient increased rapidly for sliding less than $3.5 \mathrm{~mm}$, suggesting that the macroscopic contact state of fault surfaces was not stable. I did not record AE events during this small amount of sliding distance. Hereafter, AE events that occurred for sliding distance from 3.5 to $6.5 \mathrm{~mm}$ will be called the small-sliding-events. Those that occurred for a sliding distance between $12 \mathrm{~mm}$ and $17 \mathrm{~mm}$ are called the largesliding-events.

The arrivals of $P$ - and $S$-wave first motions were read by eye. The hypocenters were determined under the constraint of $y=0$. Figure $1(\mathrm{~b})$ shows the hypocenter distribution of the small- and large-sliding-events used in the present study. The error of event location was 5-10 $\mathrm{mm}$. One of the small-sliding events and two of the large-sliding events were located out of the fault plane over the error. Since, however, $P$ - and $S$-wave arrivals of these events were clear, they were used in subsequent analyses.

Figure 2(a) shows typical waveforms observed by the broadband-type transducers. Though their hypocentral distances are almost equal to one another, the amplitudes of the small-sliding events increase more rapidly than those of the large-sliding events. This suggests that the source characteristics of the AE event changed during the sliding.

The pulse width $\left(\tau_{\mathrm{p}}\right)$, the rise time $\left(\tau_{\mathrm{r}}\right)$ and the maximum amplitude $\left(A_{P}\right)$ of the $P$-wave first motion (Fig. 2(b)) were measured to obtain an estimation of the AE source dimen- (a)

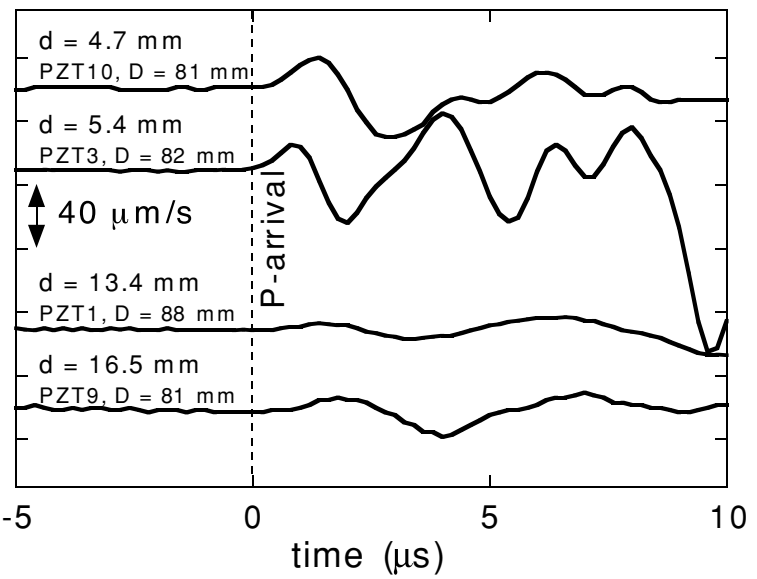

(b)

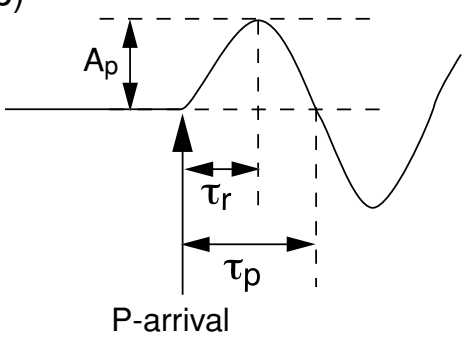

Fig. 2. (a) An example of typical waveforms. $D$ and $d$ represent the hypocentral distance and fault sliding distance, respectively at which the AE event occurred. Waveforms are aligned so that the $P$-wave arrival times are zero. (b) A schematic representation of parameters used to estimate the source characteristic.

sion (radius, $R$ ) and the magnitude of the stress drop $(\Delta \sigma)$. The waveforms obtained by the resonance-type transducer were found to be unreliable and the waveforms to be distorted by traveling in such an inhomogeneous medium as granite. Hence, only those waveforms satisfying both of the following conditions were used: (1) impulsive $P$-wave first motion was observed by the broadband-type transducers; (2) $S$ - $P$ time measured by the broadband-type transducer on which the impulsive first motion was observed was in a range 9-15 $\mathrm{s}$, which corresponds to a hypocentral distance $(D)$ of about 60 and $100 \mathrm{~mm}$, respectively. When more then one waveform from a single event satisfied the above conditions, $\tau_{\mathrm{p}}, \tau_{\mathrm{r}}$ and $A_{P}$ were measured on each waveform. The source dimension and the stress drop were then averaged following their calculation using $\tau_{\mathrm{p}}, \tau_{\mathrm{r}}$ and $A_{P}$ of individual waveforms.

The average of $\tau_{\mathrm{p}}$ with an $S-P$ time of 9-11, 11-13 and 13-15 $\mu$ s was $2.3 \pm 0.9,2.5 \pm 1.0$ and $2.6 \pm 0.7 \mu \mathrm{s}$, respectively; that of $\tau_{\mathrm{r}}$ was $1.5 \pm 0.7,1.6 \pm 0.7$ and $1.8 \pm 0.6 \mu$ s for the respective $S-P$ time ranges. Though both $\tau_{\mathrm{p}}$ and $\tau_{\mathrm{r}}$ seem to slightly increase with $S$ - $P$ time, the difference is not significant compared with their standard deviation.

Source characteristics of $R$ and $\Delta \sigma$ were estimated from the following relations based on the circular crack model of Sato and Hirasawa (1973).

$$
\tau_{\mathrm{p}}=\frac{R}{V_{\mathrm{R}}}(1-k)
$$



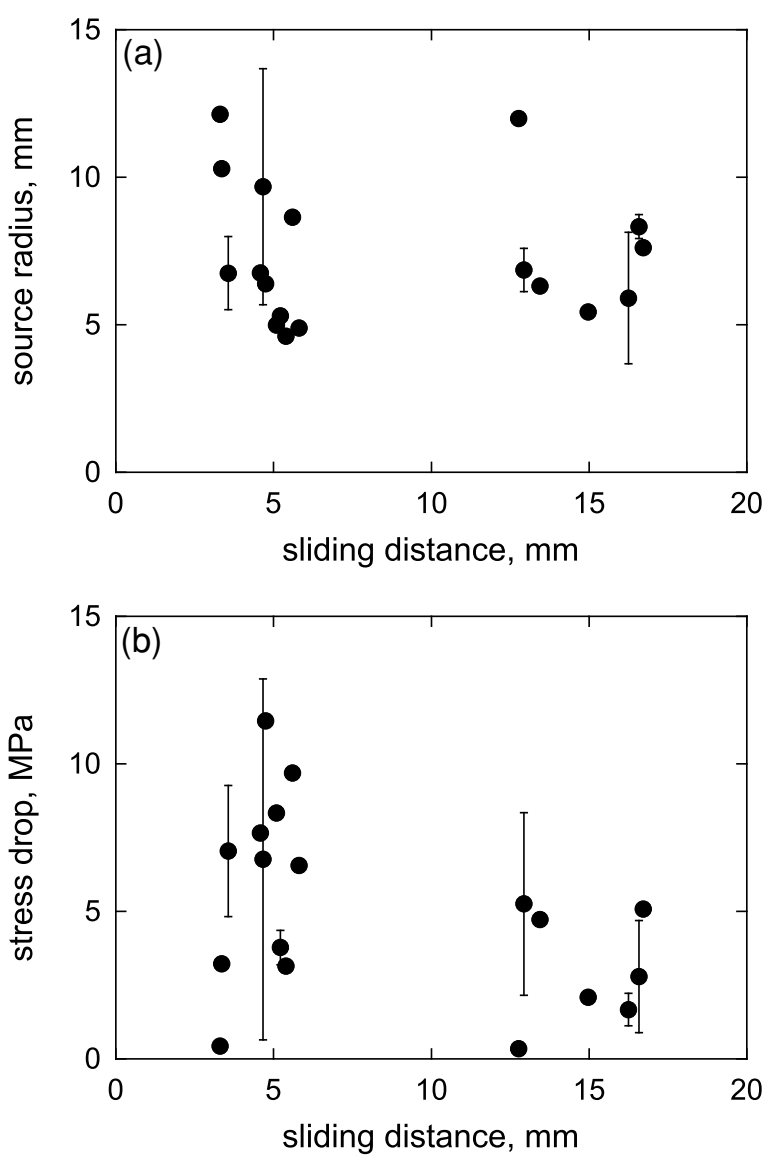

Fig. 3. Variation of (a) AE source radius and (b) stress drop with respect to sliding distance. When two or more waveforms were used to estimate source parameters of a single event, the standard error was attached.

$$
\frac{A_{P}}{\tau_{\mathrm{r}}}=\frac{96}{7 \pi} \frac{\Delta \sigma}{\mu} \frac{\left(V_{P} / V_{S}\right)^{3}}{4 \pi V_{S} D} \frac{V_{\mathrm{R}}^{2} R}{\left(1-k^{2}\right)^{2}} \sin 2 \theta \cos \phi
$$

where $V_{P}, V_{S}$ and $V_{\mathrm{R}}$ is $P$-wave velocity, $S$-wave velocity and rupture velocity, respectively; $\mu$ is the rigidity and assumed to be $30 \mathrm{GPa}$; $\theta$ denotes angle between the $y$ axis and the ray path and $k=\left(V_{\mathrm{R}} / V_{P}\right) \sin \theta ; \phi$ represents the azimuth of the ray path measured from slip direction. The $P$-wave velocity of uncompressed Oshima granite was $5 \mathrm{~km} / \mathrm{s}$. It was assumed that $V_{S}=V_{P} / 1.73$ and $V_{\mathrm{R}}=0.8 V_{S}$. The fault mechanism solutions of AE events were assumed to be the same as those expected from the macroscopic sliding. The angles of $\theta$ and $\phi$ were calculated from coordinates of transducers and hypocenters. To avoid numerical instability, data with $\phi>75^{\circ}$ were not used.

Figure 3 shows the estimated source radius and the stress drop with respect to sliding distance. The average value of $R$ was $7.3 \pm 2.5$ and $7.5 \pm 2.2 \mathrm{~mm}$ for the small- and the large-sliding events, respectively. These values are close to those reported by Yabe et al. (2003). When an outlier in the large-sliding events was excluded, the average $R$ was $6.7 \pm 1.1 \mathrm{~mm}$. The difference in source radius between the small- and the large-sliding events is not significant and, therefore, it can be said that the AE source dimension is conserved during the frictional sliding.

The estimated magnitudes of stress drop were in the same range as those of the $\mathrm{AE}$ events associated with fracture of

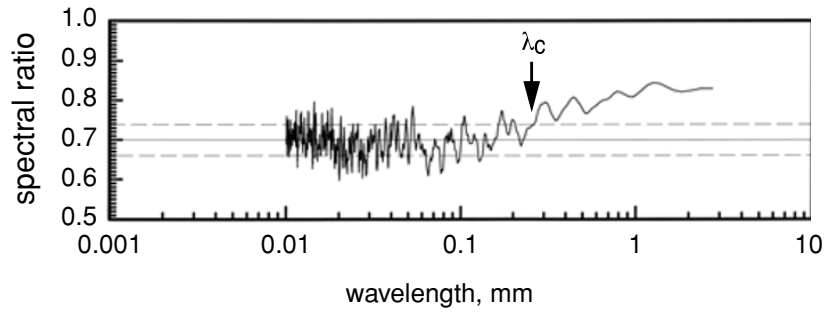

Fig. 4. Power spectral ratio of surface topography after the experiment to that before the experiment. Solid and dashed lines show the average and the standard deviation of the ratio. $\lambda_{c}$ is the upper fractal limit wavelength of the fault surface topography before the experiment.

intact rocks (Sellers et al., 2003) and natural earthquakes. The average $\Delta \sigma$ of the small- and the large-sliding events was $6.2 \pm 3.2$ and $3.1 \pm 1.9 \mathrm{MPa}$, respectively. Consequently, it can be concluded that the magnitude of stress drop is reduced significantly with sliding.

The friction coefficient was about 0.62 and 0.66 for the small- and the large-sliding distances, respectively. The average stress drops of the small- and large-sliding events were $100 \%$ and $47 \%$, respectively, of the macroscopic shear stress. These results provide evidence of a large drop in the magnitude of the stress relative to the macroscopic shear stress, suggesting that the AE events occurred in areas of stress concentration (discussed later in this article).

\subsection{Spectral analysis of surface topography}

Figure 4 represents the power spectral ratio of the fault surface topography from after the sliding to before the sliding. Spectra of 18 profiles were ensemble averaged before determining the ratio. The artificial ground surface has the upper fractal limit wavelength $\left(\lambda_{c}\right)$, which is close to the grain size of abrasive used to grind the surface (Brown and Scholz, 1985; Power et al., 1988). Topographies of wavelengths shorter than $\lambda_{\mathrm{c}}$ will be denoted here as the fractaldomain topographies, while those of wavelengths longer than the grain size $(\sim 1 \mathrm{~mm})$ will be denoted grain-scale topographies.

$\lambda_{\mathrm{c}}$ of the fault surface topography before the experiment was about $0.25 \mathrm{~mm}$. The spectral ratio of the fractaldomain topographies was about 0.7 , while that of the grainscale topographies was about 0.8 . This result indicates that the fractal-domain topographies were worn more efficiently than the grain-scale topographies. The same wavelength dependence of wear rate was observed on fault surfaces ground by a different radius abrasive.

\section{Discussion}

It is known that grain-scale convexes exist on the fault. This grain-scale convex is overlaid with asperities with a scale smaller than $\lambda_{c}$. A large AE event is considered to be generated by a coherent fracture of small-scale asperities on a grain-scale convex (Yabe et al., 2003). It is then expected that the AE source dimension is controlled by the width of the grain-scale convex. The width of convex was estimated to be about $10 \mathrm{~mm}$ on average, which is close to the $\mathrm{AE}$ source dimension. Spectral analysis of the fault surface indicated that the grain-scale topographies were not significantly worn during the sliding, suggesting that the width of 
(a)

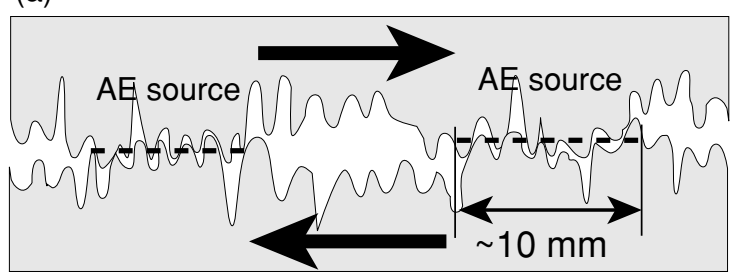

(b)

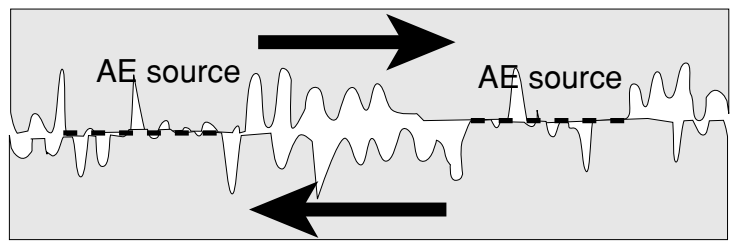

Fig. 5. Schematic illustration of contact state of faults at (a) small sliding distance and (b) large sliding distance. Thick dashed lines indicate AE source region. Large arrows represent the macroscopic sliding. See text for details.

the grain-scale convexes would be conserved. This result is supported by observation of the fault surface topographies after the experiment. As a result, it can be concluded that the AE source dimension was also conserved against wear during the sliding.

When the amplitudes of the fractal-domain topographies are large, asperities on the contacting convexes interlock with each other, as schematically illustrated in Fig. 5(a). In this case, the strength of asperity interlock should be large. It can then be expected that the stress drop of an AE event occurring on such faults should be large. Results from the evolution of spectral analysis of the fault surface topographies revealed that the fractal-domain topographies were efficiently worn during sliding. Consequently, the surface of grain-scale convex should become smooth, as illustrated in Fig. 5(b). The small-sliding events should occur on faults such as those shown on Fig. 5(a), while the large-sliding events should be generated on such faults those shown on Fig. 5(b). Hence, the reduction of the stress drop of AE events during sliding is considered to be a result of the reduction of the fractal-domain topographies during the sliding.

\section{Conclusion}

The evolutions of AE source characteristics and fault surface topography were investigated by a steady sliding experiment. It was found that the $\mathrm{AE}$ source dimension was conserved during the sliding, while the magnitude of the stress drop was significantly reduced by the sliding. Wearing of the fault surface showed wavelength dependence. The fractal-domain topographies were more efficiently worn than the grain-scale ones. These results imply that the AE source dimension is controlled by the size of grain-scale convex, while the magnitude of the stress drop depends on the amplitude of the fractal-domain asperities.

The grain-scale topographies and the fractal-domain ones played different roles in the AE source process. Since the elastic wave radiation is one of the major energy dissipation processes during frictional sliding (Yoshioka, 1986), the grain-scale and the fractal-domain topographies should also play different roles in rock friction.

Acknowledgments. Critical comments by Drs. M. Nakatani, University of Tokyo, and S. Stanchits, GFZ, Potsdam, were profitable to improve the manuscript.

\section{References}

Brown, S. R. and C. H. Scholz, Broad bandwidth study of the topography of natural rock surfaces, J. Geophys. Res., 90, 12,575-12,582, 1985.

Power, W., T. E. Tullis, and J. D. Weeks, Roughness and wear during brittle faulting, J. Geophys. Res., 93, 15,268-15,278, 1988.

Sammonds, P. and M. Ohnaka, Evolution of microseismicity during frictional sliding, Geophys. Res. Lett., 25, 699-702, 1998.

Sato, T. and T. Hirasawa, Body wave spectra from propagating shear cracks, J. Phys. Earth, 21, 415-431, 1973.

Sellers, E. J., M. O. Kataka, and L. M. Linzer, Source parameters of acoustic emission events and scaling with mining-induced seismicity, J. Geophys. Res., 108, doi:10.1029/2001JB000670, 2003.

Weeks, J., D. Lockner, and J. Byerlee, Change in b-values during movement on cut surfaces in granite, Bull. Seismol. Soc. Am., 68, 333-341, 1978.

Yabe, Y., Rate dependence of AE activity during frictional sliding, Geophys. Res. Lett., 29, doi:10.1029/2001GL014369, 2002.

Yabe, Y., N. Kato, K. Yamamoto, and T. Hirasawa, Effect of sliding rate on the activity of acoustic emission during stable sliding, Pure Appl. Geophys., 160, 1163-1189, 2003.

Yoshioka, N., Fracture energy and the variation of gouge and surface roughness during frictional sliding of rocks, J. Phys. Earth, 34, 335$355,1986$.

Y. Yabe (e-mail: yabe@aob.geophys.tohoku.ac.jp) 